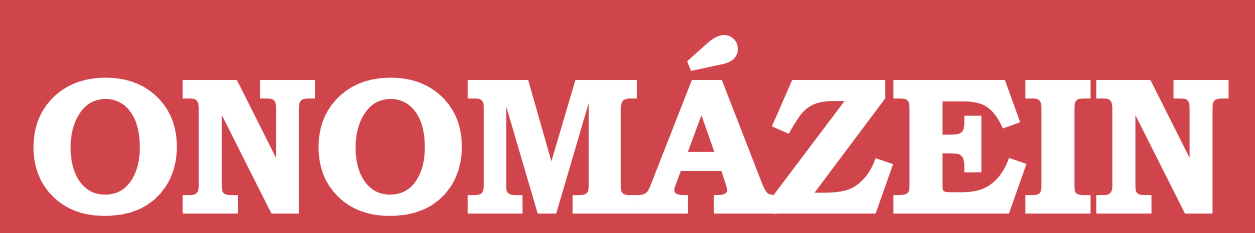

Revista semestral de lingüística, filología y traducción
PONTIFICIA UNIVERSIDAD CATÓLICA DE CHILE CATOLICA DE CHILE
FACULTAD DE LETRAS

\title{
La fraseología en la clase de lengua extranjera: ¿misión imposible?
}

Phraseology in the foreign language classroom. Mission impossible?

\section{Larissa Timofeeva Timofeev}

Universidad de Alicante

España

\section{(C) (i) $\Theta$}

Larissa Timofeeva: Departamento de Filología Española, Lingüística General y Teoría de la Literatura, Universidad de Alicante. España. Correo electrónico: timofeeva@ua.es 


\section{Resumen}

En este artículo ofrecemos una perspectiva diferente del aprendizaje y la enseñanza de la fraseología en clase de lengua extranjera (LE). Partiendo de la premisa sobre el carácter eminentemente holístico del lenguaje, concebimos las unidades fraseológicas (UF) como elementos habituales e inherentes en la comunicación verbal, por lo que no pueden excluirse del proceso de adquisición de la competencia comunicativa en una LE. Nuestra propuesta consiste en partir de la fraseología para optimizar el desarrollo de diversas subcompetencias de la competencia comunicativa. Para ello, resulta imprescindible indagar en la configuración y el funcionamiento del significado fraseológico, que, según defendemos en este trabajo, conforma un conjunto de informaciones de diversa índole que se articulan en dos niveles, el semántico y el pragmático. Tal indagación permite desarrollar una serie de consideraciones con interesantes implicaciones didácticas.

Palabras clave: fraseología; aprendizaje y enseñanza de lenguas extranjeras.

\section{Abstract}

This article presents a different perspective on the learning and teaching of the phraseology in the foreign language class. Considering the holistic nature of language, we conceive phraseological units as completely common and inherent elements in verbal communication; that is why we cannot exclude them from the acquisition of the communicative competence. In this process we propose as a starting point the phraseology for developing the different parts of communicative competence. Thus, it is essential to look into the phraseological meaning, its configuration and its working. As we explain in this article, phraseological meaning is conformed by several informational chunks articulated in two levels, the semantic and the pragmatic. These considerations have interesting teaching implications.

Keywords: phraseology; foreign language learning and teaching. 


\section{Introducción ${ }^{1}$}

Uno de los consabidos escollos con los que se encuentran los profesores de cualquier lengua extranjera (LE) es la enseñanza de la fraseología. Todos los idiomas cuentan con un buen número de expresiones que, frente a un funcionamiento discursivo asimilable al de un lexema, presentan una riqueza semántica difícilmente representable a través de una sola palabra. Tales expresiones, o unidades fraseológicas (UF), poseen una serie de características propias pero, por otro lado, forman parte de una dinámica holística común y natural en las lenguas, lo que las convierte en recursos lingüísticos completamente corrientes. Por ello, el aprendizaje de una lengua incluye el aprendizaje de su fraseología, proceso cuyo diseño didáctico ha de ir precedido, a nuestro juicio, por una reflexión sobre las particularidades de la significación fraseológica y su funcionalidad comunicativa. Esta es la tarea que pretendemos abordar en este artículo.

Empezaremos, por tanto, por un planteamiento inicial sobre el papel de la fraseología en la comunicación lingüística y sus implicaciones para el ámbito de la enseñanza de LE. Las reflexiones en torno a estos aspectos nos llevarán a presentar un modelo de significación fraseológica articulado en dos niveles que, a nuestro juicio, consigue reflejar el abanico de valores que lo configuran (Timofeeva, 2012). En este sentido, y habida cuenta del papel de la fraseología en la comunicación lingüística, parece apropiado partir del modelo de significación fraseológico para la formación de ciertos aspectos de la competencia comunicativa en una LE. Por ello, en la parte final del trabajo, el lector encontrará algu- nos esbozos metodológicos en los que se fundamentaría el diseño de materiales docentes destinados a estimular en el estudiante una reflexión metalingüística sobre el significado y el valor discursivo que representa el uso de UF. No se trata de actividades ni de ejercicios concretos, cuya elaboración sobrepasa los objetivos de este trabajo, sino de orientaciones y reflexiones iniciales sobre la aplicación didáctica de las ideas aquí desarrolladas.

\section{2. ¿Por qué aprender la fraseología?}

En nuestra actividad comunicativa diaria, casi en la totalidad de acciones y situaciones relacionadas con el uso del lenguaje, recurrimos a las UF². El discurso de un hablante nativo de una lengua está plagado de combinaciones lexemáticas más o menos adscribibles al universo fraseológico, pues las estructuras prefabricadas se erigen en nuestras estrategias para salvar lo que Levinson (1995: 95-98; 2004 [2000]: 29) denomina el problema de "cuello de botella". En efecto, el proceso de la transmisión de datos que constituye la comunicación se ve considerablemente lentificado por las características de nuestro aparato articulatorio, lo cual produce un desfase entre la celeridad del pensamiento y la lentitud de la pronunciación. La solución a este problema que ha adoptado el sistema cognitivo humano consiste en asignar determinados valores inferenciales a ciertas estructuras lingüísticas. De este modo, procesamos una parte de información por defecto, en asociación a determinadas circunstancias lingüísticas y contextuales, Io que aminora el impacto del citado desfase en nuestra actividad comunicativa.

1 Queremos agradecer a las doctoras Leonor Ruiz Gurillo y M. ${ }^{a}$ del Mar Galindo Merino la lectura de la versión inicial de este artículo y todas sus correcciones y sugerencias.

2 Unidad fraseológica es la denominación más aceptada en los ámbitos de distintas lenguas para referirse a las manifestaciones lingüísticas objeto de estudio de la fraseología. Según la definición ya clásica de Corpas (1996: 20), son "unidades léxicas formadas por más de dos palabras gráficas en su límite inferior, cuyo límite superior se sitúa en el nivel de la oración compuesta. Dichas unidades se caracterizan por su alta frecuencia de uso, y de coaparición de sus elementos integrantes; por su institucionalización, entendida en términos de fijación y especialización semántica; por su idiomaticidad y variación potenciales; así como por el grado en el cual se dan todos estos aspectos en los distintos tipos". El alcance del término, por tanto, es muy amplio y abarca todas aquellas combinaciones de palabras a las que la tradición lingüística española se ha referido con los nombres de modismos, frases hechas, expresiones fijas, así como proverbios, refranes, etc. 
Desde pequeños asimilamos este holismo del lenguaje ${ }^{3}$ como parte del comportamiento sociolingüístico. Interiorizamos los diversos procedimientos léxicos y gramaticales que permiten obtener mayores efectos perlocutivos a través de estructuras compactas. Aprendemos, con mayor o menor suerte, a construir textos cuya eficacia y eficiencia se mide también, entre otras cosas, en relación a su economía. En definitiva, nuestro éxito comunicativo depende en importante medida del grado de conocimiento de los mecanismos holísticos del lenguaje y de la habilidad de ponerlos a nuestro servicio (cfr. Pawley y Syder, 1983)

Las UF conforman una parte sobresaliente de tales mecanismos. Se insertan en el discurso de manera integradora asegurando su fluidez y naturalidad. No en vano, estudios empíricos demuestran que el discurso de un hablante nativo consigue su agilidad y precisión gracias al uso de UF (cfr. Lennon, 1998; Lee, 2004; Wood, 2004; Prodromou, 2008)5. Pero ¿qué sucede con los alumnos de lengua extranjera? ¿Cómo conseguir que su discurso se asemeje en términos comunicativos al de un nativo? ¿Por qué es imprescindible que los fraseologismos participen en este proceso formativo? ¿Cómo podemos facilitar al alumno la comprensión y la asimilación de la complejidad fraseológica?
Lennon (1998) indica que el discurso de un hablante extranjero a menudo se caracteriza como "no idiomático", cuestión que a ojos de un nativo más que con la corrección se relaciona con la aceptabilidad, una noción algo confusa que define la reacción de cierta extrañeza ante lo dicho por un extranjero (Lennon, 1998: 11):

... the term "non-idiomatic" is sometimes used to designate non-nativelike discourse, where the non-nativelike quality cannot be pinned down to lexical choice error at the lexeme level, nor to grammatical error. In other words, the discourse is well-formed and is lexico-semantically feasible. Non-idiomatic discourse is non-nativelike rather than erroneous. Native speakers might react to it with the comment "It's not wrong, but you don't say it".

Dicho de otro modo, cabe entender que el aprendizaje de una LE, así como la integración social y cultural de un extranjero en la comunidad lingüística de acogida, pasa también por la integración "fraseológica", es decir, la incorporación de las UF a su discurso6.

El acuerdo, común y unánime hoy en día, sobre la imprescindibilidad de los contenidos fraseológicos en una clase de LE queda reflejado tanto en el Marco común europeo de referencia para las lenguas (MCER) como en numerosos trabajos sobre el tema escritos en los últimos años

3 El holismo se entiende aquí, por tanto, en su concepción gnoseológica según la cual el significado de un todo no es equivalente a una mera suma de las partes que lo componen, por lo que se tiene que contemplar desde una perspectiva integradora. Tal visión conlleva que partiendo de un todo podemos entender y explicar el comportamiento de sus partes, pero no al revés

4 Pawley y Syder (1983), en un trabajo de obligada referencia para el tema, muestran cómo el holismo lingüístico, expresado especialmente a través de la fraseología, fundamenta el nivel de soltura en el uso de una LE. Aunque muchas de las ideas de Pawley y Syder requieren de actualización teórica, la propuesta que aquí desarrollamos se inspira claramente en algunos de sus planteamientos.

5 Prodromou (2008: 61-62), recogiendo las palabras de Altenberg y Eeg-Olofsson, lo expresa de una manera muy gráfica al decir que "prefabricated expressions of all kinds are widely considered to be "a kind of "autopilot" which the speaker can switch on to gain time for the creative and social aspects of the speech process' (Altenberg and Eeg-Olofsson, 1990: 2)". Se trata de una cita especialmente reveladora pues subraya la función creativa y social de las UF, cuestión que retomaremos al presentar nuestra visión de la significación fraseológica.

6 No obstante, la integración "fraseológica" no resulta un camino de rosas para un hablante no nativo, no tanto por los motivos lingüísticos sino por los sociales. A veces, el uso de ciertos procedimientos fraseológicos puede suponer un fracaso pragmático para un extranjero, como es el caso de locuciones que se refieren a realidades culturales específicas, únicas que suscitan asociaciones connotativas percibidas como exclusivas por y para la comunidad en cuestión (v. g., dar el paseo [a alguien], 'en la Guerra Civil de 1936: Ilevárselo por la fuerza y matarlo sin juicio previo', DFDEA), y también el de las UF desautomatizadas que comentaremos sucintamente al final de este artículo (cfr. Dobrovol'skij, 1997, 1998; Prodromou, 2003: 46). 
(cfr. Prodromou, 2008: 3-90 para el inglés u Olímpio, 2006: 2-73, en español). Así, el MCER (2002: 106), en referencia a la competencia léxica, recoge de manera extensa los diversos elementos adscribibles claramente a la fraseología, si bien no utiliza este término, ya que habla de

expresiones hechas, que se componen de varias palabras que se utilizan y se aprenden como un todo. Las expresiones hechas incluyen: fórmulas fijas [...]; refranes, proverbios, etc. [...]; modismos [...]; metáforas lexicalizadas, semánticamente opacas [...]; estructuras fijas, aprendidas y utilizadas como conjuntos no analizados, en los que se insertan palabras o frases para formar oraciones con sentido..

Vistos así, los contenidos fraseológicos están presentes en clase de LE desde los niveles iniciales y su uso activo y coherente constituye uno de los criterios (auto)evaluativos del nivel de referencia C2 (cfr. MCER, 2002: 30-31). Tal situación ha propiciado que se hable de competencia fraseológica como parte integrante de la competencia comunicativa y que esa generalmente se conciba como "el hecho de conocer las diferentes UFs de una lengua, y saber interpretarlas e integrarlas en un discurso propio, oral o escrito, adecuado según el cotexto, el contexto, la relación con el interlocutor, las normas sociales y nuestros propósitos de interacción" (Solano, 2004: 411).

No obstante, y pese a las consideraciones aducidas, adviértase que puede parecer algo incoherente afirmar el carácter esencialmente holístico de la comunicación lingüística y destacar la competencia fraseológica como un subnivel de la competencia comunicativa. A nuestro modo de ver, tal vez sea más adecuado concebir la competencia holística como un paraguas o un principio original y básico, que acoge las diversas subcompetencias configuradoras de la competencia comunicativa, que es, a fin de cuentas, el objetivo hacia el que se orienta todo proceso de aprendizaje. Decimos todo esto sin ánimo de entrar en deliberaciones teóricas, posiblemente vacuas de cara a las aplicaciones didácticas; tan solo pretendemos insistir en que la fraseología en sentido amplio es inherente y natural a cualquier lengua, por lo que cabe plantearse su "desmitificación" y asumir su aprendizaje desde esa naturalidad.

Sea como fuere, queda patente la conciencia de la necesidad de introducir los contenidos fraseológicos en clase de LE, pero también, a nuestro modo de ver, se percibe una confusión en lo que se refiere a las ya proverbiales dificultades que supone tal tarea. Todos Ios trabajos sobre el tema coinciden en subrayar este aspecto o, en palabras de Olímpio (2006: 6):

Hay [...] un consenso al respecto: todos los investigadores opinan que las UF son difíciles de enseñar y que a cualquier aprendiz de una LE le cuesta dominarlas. Algunos autores, incluso, al advertir el componente cultural inherente a las UF y al admitir la estrecha relación entre la competencia nativa y la cultura nativa, llegan a afirmar que es imposible una adquisición completa de ellas.

Las reflexiones sobre los factores causantes de un panorama tan desalentador (cfr. Olímpio, 2006: 6-14) no consiguen aclarar las cosas, ya que las causas aducidas, múltiples y heterogéneas, admiten la posibilidad de una lectura opuesta. Dicho de otro modo, las diversas características de las UF que se presentan como fuentes de su complejidad didáctica pueden verse, a la vez, como potentes mecanismos de aprendizaje que deberían facilitar el proceso. Por otra parte, en el fondo, las dificultades tradicionalmente presentadas como inherentes a las UF no resultan sustancialmente distintas a las de otras estructuras lingüísticas ( $v$. g., defectividad del paradigma, particularidades morfosintácticas, etc.) que no generan, sin embargo, tantos obstáculos de aprendizaje en esos otros casos.

Varias investigaciones y experiencias didácticas apuntan a que la raíz del "problema fraseológico" se encuentra en la fuerte vinculación que existe entre el significado de una UF y el bagaje 
cultural compartido por los hablantes nativos de una lengua que es adquirido y desarrollado desde la infancia. Esta es la principal causa que, según algunos, convierte la adquisición de la competencia fraseológica en una tarea casi imposible para un extranjero (cfr. Dobrovol'skij, 1997, 1998; Dobrovol'skij y Piirainen, 2005: 244 y sigs.; Prodromou, 2008: 61-70). Sin embargo, si el componente cultural constituye uno de los puntos clave del proceso de aprendizaje de una lengua extranjera7, cabría contemplar las UF no como un problema, sino como una solución, pues a través de ellas podemos tener acceso a muchos aspectos culturales para tratar en clase.

De esta forma, y parafraseando a Ruiz GuriIlo (2001: 21), ya que los caminos para llegar al significado de una UF "están llenos de escollos y de dificultades de interpretación”, indaguemos antes en los vericuetos de nuestra ruta para poder exponerla con claridad a nuestros alumnos. Creemos que el camino pasa por una reflexión metalingüística previa en torno al significado fraseológico, esto es, sobre aquellas particularidades semánticas y pragmáticas que hacen al hablante preferir la UF en su discurso. Tal reflexión ha de trasladarse adecuadamente al alumno para que este sea capaz de entender, al menos en parte, la actuación comunicativa de un hablante nativo al elegir un fraseologismo. Hasta cierto punto, el camino en este caso se hace al revés, puesto que un nativo realiza, si se da la ocasión, una reflexión metalingüística sobre el significado de una UF a posteriori, ya que asimila toda la gama de valores de diversa índole que lo configuran de forma paulatina y natural, como resultado de su experiencia comunicativa diaria.

En definitiva, podemos recapitular todo lo dicho hasta ahora a través de las siguientes ideas:

- El lenguaje utiliza profusamente los diversos procedimientos holísticos para salvar el problema de "cuello de botella" que se genera por el desfase entre la velocidad del pensamiento y la lentitud del sistema articulatorio. Por tanto, el holismo de las estructuras lingüísticas no es una anomalía, sino una característica inherente a la comunicación verbal.

- Las UF, que constituyen una de las manifestaciones del holismo lingüístico, se presentan, por tanto, como procedimientos lingüísticos naturales e imprescindibles, por lo que no solo es necesaria su incorporación a la clase de $L E$, sino que, de hecho, resulta inevitable.

- Es inevitable porque es el origen y la base de nuestra capacidad de comunicación y, por consiguiente, es el punto de partida del proceso de conformación de la competencia comunicativa, bien sea en la lengua materna, bien en la extranjera. Un hablante competente comprende y asimila el hecho de que cualquier lengua es idiomática en esencia (cfr. Pawley y Syder, 1983; Sinclair, 1987, 1991), ya que, de lo contrario, no podría comunicarse eficientemente.

- Si asumimos la anterior afirmación, cabe plantear el proceso de adquisición de la competencia comunicativa en una LE desde su condición holística y, por ende, podemos recurrir a las UF como punto de partida para la enseñanza de las diversas subcompetencias comunicativas. Para tal fin, deberíamos indagar previamente en la significación fraseológica para llegar, a través de ella, a los distintos aspectos de la competencia comunicativa.

Esta será nuestra tarea en los apartados que siguen.

7 Las diversas propuestas de adaptación de la noción de competencia comunicativa (Hymes, 1971) al ámbito de aprendizaje y enseñanza de LE reflejan explícitamente la importancia del componente cultural, como se puede comprobar en Llobera y otros (1995), donde se recopilan varios trabajos ya clásicos sobre el tema, así como en otras propuestas, como, por ejemplo, la de Alcón (2002) o la de Celce-Murcia (2007). Por su parte, en el MCER (2002: 10-14) se habla de la competencia comunicativa, modelada en torno a los componentes lingüístico, sociolingüístico y pragmático, como parte integrante de las competencias generales del individuo. 


\section{En torno al significado fraseológico}

Para comprender el papel comunicativo de las UF, debemos plantearnos una serie de cuestiones que nos lleven a indagar en los factores que fundamentan dicho papel. En primer lugar, cabe preguntarnos cuál es la función básica de las UF. En segundo lugar, cómo se configura esta función y qué consecuencias aplicadas puede tener tal configuración.

Como se afirma en Telija (1996: 75), el punto de partida en este recorrido debe ser la concepción de los fraseologismos como unidades denominativas. En efecto, las UF surgen como respuesta ante la incapacidad del hablante de denominar una realidad, sentimiento, acción, etc., a través de los lexemas simples disponibles. El camino elegido, en este caso, es de índole metafórica, pues básicamente se recurre a los "moldes" semánticos ya existentes que experimentan una reinterpretación del conjunto con la consiguiente adquisición de un nuevo significado.

Por tanto, la función primigenia de las UF es la denominación, cuya realización se da a través de una serie de informaciones de diversa índole que configuran el significado fraseológico completo. Pese a su indudable complejidad, creemos que es posible sistematizar muchos de los aspectos que lo conforman, lo cual proporciona pautas para una reflexión metalingüística en torno al significado de una UF.

Como se afirma en Timofeeva (2012: 203256), las UF realizan su función denominativa en dos niveles, al igual que cualquier unidad de denominación primaria ${ }^{8}$. En el primer nivel, llamémoslo semántico, el significado fraseológico representa un conjunto de bloques informativos que permiten describir los distintos aspectos de su configuración como resultado de un proceso pragmático identificable con patrones gramaticalizadores (cfr. Traugott y Dasher, 2002; Levinson, 2004 [2000]). Los bloques informativos en cuestión funcionan, fundamentalmente, como informaciones inferidas, esto es, como implicaturas que presentan diverso grado de convencionalización. Dicho grado tiene consecuencias directas en el segundo nivel de significación fraseológica, donde nos interesa el comportamiento discursivo de las UF. En efecto, recordemos que, pese a su complejidad semántica y estructural, los fraseologismos son unidades denominativas y, por tanto, funcionalmente son lexemas. Como tales, una vez insertas dentro de un enunciado mayor, las UF pueden desplegar una serie de significados adicionales de índole implicatural, en mayor o menor medida condicionados por las circunstancias contextuales.

Veamos ahora con más detalle el contenido de cada uno de los niveles descritos. La semántica fraseológica, como hemos dicho, es sistematizable a través de una serie de bloques informativos que componen el modelo macrocomponencial, inicialmente desarrollado y descrito por Telija (Telija y otros, 1990; Telija, 1996; Kabakova, 2002; Timofeeva, 2012: 205-244). Según dicho modelo, podemos destacar seis tipos de información, que exponemos a continuación.

El primer bloque recoge la información denotativa, que indica el referente de la UF. Por ejemplo, la locución meter la pata se refiere a "ser inoportuno" (cfr. DFDEA, DRAE, DUE, DFEM); esto es, la información que refleja, normalmente,

8 Las unidades figurativas en general, y las fraseológicas en particular, son de denominación secundaria "pues su significado se construye por vía de la adición de datos y matices a una unidad de denominación primaria. Por todo ello, las unidades figurativas no son semánticamente igualables a las unidades de denominación primaria, pero guardan relación con ellas en términos de subordinación" (Timofeeva, 2012: 161). Esto permite a Dobrovol'skij y Piirainen (2005: 5-18) establecer como uno de los criterios de la pertenencia de una combinación léxica al acervo fraseológico el cumplimiento del requisito de denominación adicional, esto es, de ser una manera alternativa de denominar un concepto, aun teniendo en cuenta todas las matizaciones pertinentes. 
la entrada lexicográfica de una UF9. No obstante, la descripción lexicográfica deja a menudo sin atención otras informaciones imprescindibles para comprender el significado de una UF y que exponemos seguidamente.

El segundo bloque del modelo macrocomponencial, llamado motivacional, describe el componente de imagen de la UF que representa el elemento nuclear de la semántica fraseológica. Se trata del puente cognitivo que fundamenta el salto entre la lectura primaria y el significado convencionalizado de la expresión. Adviértase que, pese a que en muchos casos se trata de operaciones metafóricas que enlazan la lectura literal y la figurativa de los componentes de la UF, el componente de imagen también puede remitir a asociaciones de otra índole, e incluso a una primera ocasión en la que el hablante oyó el fraseologismo y fue capaz de inferir su significado. Este podría ser el caso de meter la pata para muchos aprendices extranjeros; por eso la creación de una red asociativa alrededor de una UF nueva se convierte en clave para la correcta configuración del componente de imagen.

Por otra parte, este último aspecto resulta muy interesante ya que nos indica que los lazos motivacionales presentan una fuerte subjetividad, lo cual supone que el hablante puede realizar inferencias con cierto grado de variación del significado hasta llegar a establecer una etimología apócrifa de la expresión. Ello explica por qué algunas UF experimentan cambios semánticos importantes fundamentados por el "olvido" de la etimología original.

Los conocimientos implicados en la construcción del componente de imagen de una UF son muchos y muy heterogéneos, pues incluyen informaciones conceptuales, históricas, culturales, etc. (cfr. Timofeeva, 2012: 173-197, 211-222).
Asimismo, resulta prácticamente imposible encontrar tipos "puros", ya que en la mayoría de los casos los lazos motivacionales de diversa naturaleza actúan e interactúan en la configuración del componente de imagen. Lo que parece claro es que para el hablante una UF siempre está motivada, ya que en ello radica el porqué de la elección de un fraseologismo frente a un lexema libre dentro de una cadena discursiva.

El tercer bloque informativo del primer nivel de significado fraseológico hace referencia al aspecto evaluativo, concretamente a la evaluación racionalizada que encierra el significado de una UF. En este sentido, la evaluación se articula en dos ejes, el cualitativo y el cuantitativo, donde la información se distribuye entre los puntos de negativo - neutral - positivo y menos de la norma - la norma - más de la norma, respectivamente. Así, meter la pata parece mostrar cierta tendencia hacia el polo negativo, cualitativamente hablando, y más de la norma (i. e., se pondera el grado de la inoportunidad), en lo que a la evaluación cuantitativa concierne. Recuérdese que son informaciones evaluativas de primer nivel que, en función del grado de su convencionalización, pueden experimentar ciertas modificaciones cuando se inserten dentro de un enunciado mayor.

El siguiente bloque que conforma el modelo macrocomponencial del primer nivel de significado fraseológico también alude a la información evaluativa, pero esta vez en términos de valoración emotivo-emocional. Concretamente, nos referimos aquí al sentimiento-actitud del que, por una parte, dota el hablante a la UF al introducirla en su discurso y que, por otra, pretende "infundir" en su oyente. Dicho de otro modo, para los hablantes el significado de una UF incluye una gama de sentimientos-actitudes concretos, por lo que su uso permite transmitir

9 En relación al bloque denotativo hay que señalar también otro aspecto interesante, y es que la denotación fraseológica siempre contiene una referencia cualitativa; esto es, una UF no solo denota su referente, sino que también lo caracteriza, y ello es extensible incluso a aquellas UF cuya función básica es la identificación, ya que su referente es único e individual. Así, la locución caballero de la triste figura no solo identifica a Don Quijote, sino que lo caracteriza. Para más detalle sobre esta cuestión vid. Dobrovol'skij (1990), Timofeeva (2012: 206-211). 
de una forma "compacta" un conjunto muy rico de informaciones de tal índole. Por otro lado, el hablante pretende que su oyente experimente los mismos sentimientos-actitudes y actúe en consecuencia. De esta manera, el bloque de información emotivo-emocional adquiere una especial relevancia en relación al efecto perlocutivo que busca conseguir el hablante en su oyente. Adviértase que tal efecto se culmina ya en el segundo nivel de significación fraseológica, pues las circunstancias contextuales juegan aquí un papel decisivo. Asimismo, es evidente, en este sentido, la estrecha relación que existe entre este bloque informativo y el de evaluación racionalizada, ya que una valoración cuantitativa y cualitativa incide directamente en la emotivo-emocional. También el bloque motivacional determina en importante medida las características del sentimiento-actitud que encierra la UF, puesto que la imagen que sustenta el significado fraseológico a menudo contiene elementos que proporcionan las claves de ello ${ }^{10}$, si bien resulta importante subrayar la fuerte raigambre cultural de todos estos aspectos. Ilustrando lo dicho con la locución meter la pata, podemos ver que al elegir esta UF el hablante pretende al menos resaltar la intensidad de la equivocación cometida y que su oyente comprenda y comparta dicho sentimiento. Obviamente, las circunstancias contextuales del enunciado mayor en el que se inserte la UF (i. e., el segundo nivel) perfilarán si tal intensidad se refiere, por ejemplo, al grado de arrepentimiento del agente de la acción de meter la pata o de algún otro sentimiento.

El quinto bloque del modelo de significación fraseológica de primer nivel alude a la información estilística, esto es, a la adecuación discursiva de la UF. En efecto, y como la mayoría de los diccionarios suele indicar, los fraseologismos se adscriben a diversos registros, desde los más coloquiales a los más formales, por lo que esta información resulta imprescindible para la correcta comprensión del significado de una UF. Así, en el caso que analizamos aquí, meter la pata se adscribe al registro informal o coloquial (vid. DFDEA, DUE, DRAE), por lo que se adecua a contextos discursivos correspondientes. Es interesante observar, sin embargo, que los datos estilísticos constituyen un potente mecanismo para generar fácilmente en el segundo nivel de significación diversos efectos discursivos, como el humor o la ironía, a través de la desautomatización de los valores fijados (cfr. Timofeeva, 2009a, 2009b).

Por último, el sexto bloque del modelo recoge datos sobre el comportamiento gramatical de Ia UF. Como bien se sabe, tradicionalmente la fraseología utiliza la información gramatical como punto de partida de la descripción del significado; de ahí que los fraseologismos aparecen fijados en los diccionarios como locuciones nominales, adjetivas, verbales, etc. No obstante, una somera reflexión al respecto muestra que tal información solo revela las características formales, pero no consigue reflejar plenamente las particularidades funcionales de las UF. Por ello, como señalaba en su ya clásico trabajo G. Wotjak (1983), pese a una estrecha relación que existe entre la forma y la función, no podemos mezclarlas, pues un análisis funcional no puede partir de una descripción formal, basada, en la mayoría de los casos, en la función gramatical del primer elemento de la UF. Por tanto, un modelo de significación fraseológica que aquí presentamos recoge la información gramatical como ciertamente periférica, cuya importancia se mide en relación a su valor semántico. En otras palabras, asimilamos básicamente las ideas de la lingüística cognitiva según las cuales un valor gramatical es semántico en esencia (cfr. Cifuentes, 1994, 2003; Cuenca y Hilferty, 1999; Levinson, 2004 [2000]: 398 y sigs.; Dobrovol'skij y Piirainen, 2005), ya que re-

10 Vid. en Timofeeva (2012: 227-228) el análisis de la locución echar sapos y culebras, donde los elementos sapo y culebra influyen de manera directa en el sentimiento-actitud de rechazo y animadversión que recoge el bloque de evaluación emotivo-emocional de esta UF. 
sulta evidente que la categoría gramatical de la UF nos orienta sobre si se trata de una acción, un objeto, una cualidad, etc. Así, meter la pata, que formalmente es un verbo, alude a ese aspecto accional acorde con su forma gramatical. Esta información representa un valor especial para el tratamiento interlingüístico de las UF, como por ejemplo la traducción, ya que el traductor ha de tener en cuenta la información gramatical de la UF original para suplirla, si es necesario, a través de diversos medios en el texto meta.

Los seis bloques del modelo de primer nivel actúan, obviamente, de forma simultánea e interactúan estrechamente entre sí (vid. Timofeeva, 2012: 236-240). El esquema 1 refleja gráficamente estas relaciones.

\section{ESQUEMA 1}

El modelo macrocomponencial del significado fraseológico de primer nivel

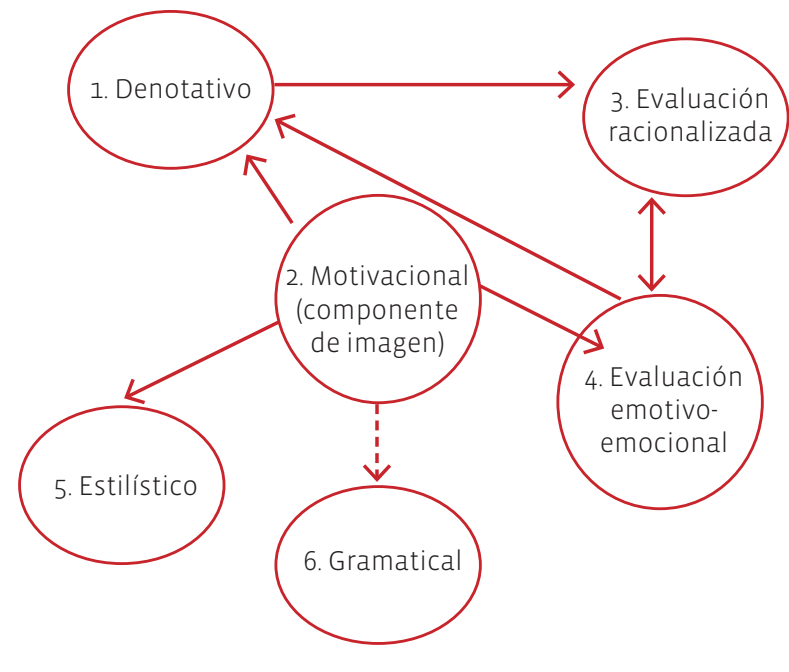

Como se puede apreciar de la explicación del modelo macrocomponencial, las informaciones que lo configuran, especialmente las de índole evaluativa y estilística, se presentan como implicaturas con diverso grado de convencionalización. Tal grado influye en el comportamiento discursivo de la UF, esto es, cuando esta empieza a funcionar como un lexema dentro de un enunciado mayor. Así, cuanto mayor sea el grado de convencionalización de los valores implicatura- les de primer nivel, mayor será su influencia en el discurso; mientras que si ese grado es menor el contexto puede modificar o cancelar dichos valores de primer nivel. En nuestro ejemplo de la UF meter la pata parece que la información ponderativa de la inoportunidad cometida representa un grado alto de convencionalización, por lo que no solo permanecerá básicamente inalterada en el discurso, sino que influirá en él y lo "teñirá" en tales términos. No sucede lo mismo con la evaluación cualitativa, pues la tendencia hacia el polo negativo presenta menor nivel de convencionalización y, por tanto, puede verse modificada por las circunstancias del contexto. Relacionado con ello, el sentimiento-actitud que el hablante pretende transmitir a su oyente preservará el valor ponderativo, pero que la acción descrita por meter la pata se evalúe como positiva o negativa dependerá en mayor medida del contexto discursivo concreto. Asimismo, la información estilística que identifica meter la pata como coloquial muestra un grado de convencionalización muy alto, lo cual indica que su presencia en el discurso va a dotar a este de tal valor coloquial. Adviértase que si utilizamos la UF en cuestión en un registro distinto, estaríamos hablando ya de una muestra de desautomatización discursiva, esto es, un uso estilísticamente inadecuado de una UF para conseguir determinados efectos, fundamentalmente humorísticos (Timofeeva, 2009a).

Recapitulando lo expuesto, observamos que el significado de una UF, aun siendo indudablemente complejo, es sometible a una sistematización que permite pautar la reflexión metalingüística en torno a él. A nuestro modo de ver, tal reflexión resulta imprescindible, tanto para el profesor como para el alumno, si bien, naturalmente, con adaptaciones pertinentes. Creemos que el modelo de significación fraseológica presentado consigue que el alumno "vea”, comprenda la variedad de informaciones que encierra y aporta al discurso la UF, así como memorice el significado de una forma ordenada y más cons- 
ciente. Este último aspecto, como bien se sabe, constituye el principal caballo de batalla de la didáctica de la fraseología, que ha llevado a plantear diversas técnicas de aprendizaje de las UF (cfr., v. g., Ruiz Gurillo, 1999; Forment, 1998; Detry, 2008; Leontaridi y otros, 2008).

La visión de la significación fraseológica que aquí hemos desarrollado es cualitativamente distinta a otras propuestas y, en nuestra opinión, sienta las bases para un camino más eficaz hacia la adquisición de la competencia fraseológica y, en última instancia, la comunicativa. Por ello, en el siguiente apartado reflexionaremos sobre las posibles vías de explotación didáctica del modelo presentado.

\section{Algunas orientaciones metodológicas}

En este último apartado realizaremos unas breves orientaciones metodológicas sobre la elaboración de ejercicios de fraseología a partir de las ideas expuestas hasta ahora. Cabe señalar que se trata de unas aproximaciones iniciales, modelos generales que, sin duda, son mejorables y necesitan de una mayor elaboración. Nuestro objetivo aquí consiste solamente en ofrecer unas pautas de aplicación de nuestro modelo, que deben adaptarse para ser plasmadas, posteriormente, en tareas y materiales didácticos concretos. Sin embargo, creemos que este último cometido es de por sí muy amplio y complejo, por lo que merece un trabajo aparte. Aquí tan solo nos limitaremos a hacer unas sucintas indicaciones al respecto. Generalmente, no haremos consideraciones sobre el nivel de dificultad de las propuestas, pues creemos que la mayoría de ellas pueden ser elaboradas y matizadas de acuerdo con las circunstancias concretas del aula.

\subsection{Dibujando la fraseología}

En la línea de la "formación" del alumno en la reflexión metalingüística sobre el significado fraseológico, puede resultar interesante partir desde una representación gráfica de algunas UF, esto es, del "dibujo" del significado de una UF. Tal enfoque, asimismo, se integra dentro de la concepción multimodal, tan en boga en el ámbito de enseñanza-aprendizaje de lenguas extranjeras en la actualidad. La importancia que se le concede a la multimodalidad no busca otra cosa que intentar reflejar la realidad de la comunicación, pues en ella recurrimos a diversos "lenguajes", no solo al verbal, que combinamos para conseguir nuestros fines comunicativos de una manera totalmente natural. Como señala en este sentido Kress (2000: 338-339), "there is a semantic trade among speech, image, and writing (and other modes, too, and via other senses-touch, feel, taste) that is, simply, human. One could not know what this text is about [...], unless one looked at image and writing together".

Siguiendo esta perspectiva, podemos ofrecer a nuestros alumnos un dibujo que desgrane el significado de una UF utilizando como guía el modelo macrocomponencial expuesto. Así, empezando por una UF cuyo componente de imagen evidencia una proyección metafórica clara, conseguiremos motivar a nuestros alumnos a completar la gama de valores que engloba el significado de la unidad. Por ejemplo, la locución con las orejas gachas, que tendrá como referente (i. e., el bloque denotativo) "en estado de tristeza, frustración” (cfr. DFDEA, DRAE, DUE, DFEM), permite representar fácilmente la información motivacional a través del dibujo de un perro en actitud correspondiente de acuerdo con la metáfora que sustenta el componente de imagen de esta UF. Respecto a la evaluación racionalizada, esta locución parece mostrar una valoración “más de la norma” en el eje cuantitativo y tendencia hacia el polo negativo en el cualitativo, por lo que nuestro dibujo deberá recoger esta información. En lo que a la evaluación emotivo-emocional concierne, con las orejas gachas expresa, ante todo, un sentimiento de frustración por no haber conseguido lo que se deseaba, un sentimiento que se pondera y que se pretende que experimente el interlocutor para formar 
una determinada actitud hacia el referente, que puede ir desde la lástima y la compasión hasta el desprecio y la humillación. Es evidente que es la coyuntura contextual concreta la que se encargará de determinar tales actitudes. En el resto de los bloques se trata de una UF coloquial y que actúa sintácticamente como adverbio. En el esquema 2 recogemos una posible representación del significado de esta UF.

\section{ESQUEMA 2}

Posible representación gráfica de la locución con las orejas gachas

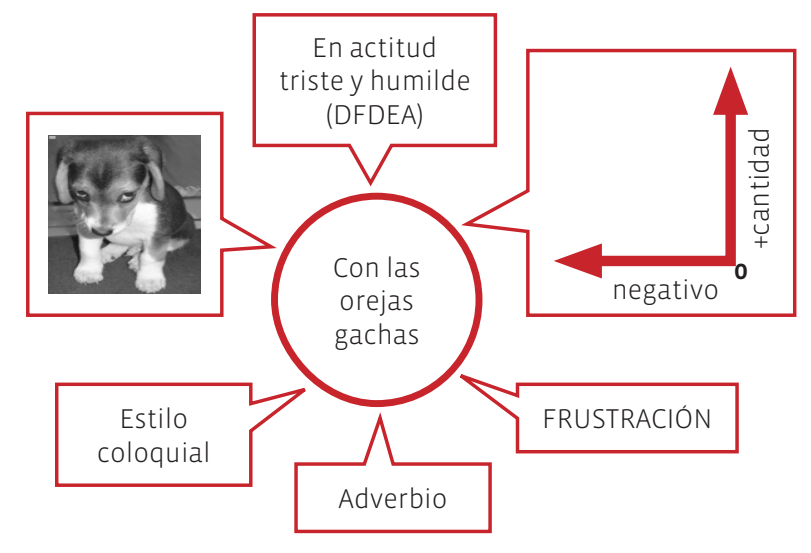

Obsérvese que el gráfico puede recurrir a las imágenes en mayor medida de la que aquí hemos hecho, pues pueden usarse para representar otras informaciones ( $v$. g., las del bloque emotivo-emocional) que en nuestro ejemplo hemos expresado a través de las palabras. Lo que resulta imprescindible es la ayuda del profesor para que explique al alumno de un modo accesible la tipología de informaciones recogidas en el gráfico, con el fin de que este cumpla su papel de elemento desencadenante del proceso de reflexión metalingüística sobre el significado de la UF en cuestión. Por tanto, consideramos que los ejercicios con los dibujos que reproducen la variedad de informaciones que configuran el significado fraseológico se muestran muy útiles, tanto para estimular la atención metalingüística como por su valor mnemotécnico.

\subsection{Dibujando la fraseología II}

Como consecuencia lógica de las actividades basadas en las orientaciones expuestas en el apartado anterior, se presenta el planteamiento de ejercicios en los que se produce el cambio de papeles, y es el alumno quien ha de buscar y elaborar un esquema del significado de las UF dadas. El papel del profesor sigue siendo clave también en esta ocasión, ya que es él quien tiene que guiar al estudiante hacia un tratamiento adecuado de todos los tipos de información que configuran el significado fraseológico completo y hacer dicho proceso atractivo y didácticamente útil.

\subsection{Las UF en el discurso}

Como hemos defendido en este trabajo, Ias UF despliegan todo su significado en dos niveles - puesto que presentan una complejidad semántica resultado de lo que Levinson (2004 [2000]) denomina intrusión de la pragmática en la semántica-y adquieren el comportamiento lexemático en cuanto se insertan en un enunciado mayor. Por tanto, es en el interior de un texto donde podemos analizar el funcionamiento completo de una UF. En este sentido, las actividades destinadas a formar la capacidad del alumno de identificar, comprender y explicar los fraseologismos dentro de una secuencia textual resultan sumamente efectivas.

De esta manera, pueden diseñarse tareas en las que al alumno se le ofrece un texto que contiene varias UF que tiene que identificar. Curiosamente, y dada la capacidad holística que se tiene en la lengua materna, los alumnos, incluso en los niveles iniciales, suelen detectar con éxito las UF en un texto adecuado a su grado de competencia, aun sin saber qué significan estas. A continuación, y guiados por el profesor y los materiales lexicográficos que se consideren oportunos, el estudiante intentará describir el significado completo de las UF siguiendo el modelo propuesto, aunque convenientemente 
adaptado según el juicio del profesor. Esto supone que, dependiendo de las características del curso, el docente explicitará en mayor o menor medida los aspectos teóricos de la configuración y el funcionamiento del significado fraseológico, haciendo hincapié en aquellos datos que resultan esenciales para promover en el alumno una reflexión metalingüística en torno a las informaciones que engloban el significado de una UF concreta y su desarrollo en el discurso. Tal reflexión llevará al discente a plantearse por qué el hablante nativo recurre a una determinada UF en un contexto dado y qué beneficios comunicativos ello aporta. Como se observa, el diseño de las actividades concretas de este tipo no solo tiene objetivos inmediatos, como la identificación, la interpretación y la explicación del significado de las UF, sino también a más largo plazo, dirigidos a la formación de la competencia metalingüística en lengua extranjera. Asimismo, como ya subrayamos anteriormente, la explicación del significado de una UF acorde al modelo propuesto le confiere una ordenación y sistematización que actúan como un procedimiento mnemotécnico, con lo cual el estudiante consigue solventar mejor las diversas aristas formales y semánticas que presentan algunas UF.

\subsection{Desautomatización fraseológica}

Quisiéramos hacer unos últimos apuntes relativos a las actividades relacionadas con la manipulación discursiva, esto es, el fenómeno de desautomatización fraseológica. Se trata de diversas alteraciones intencionadas, claramente identificables en el nivel formal, semántico o discursivo de la UF, y fácilmente reconocibles, que persiguen determinados efectos perlocutivos, especialmente de índole humorística (cfr. Ruiz Gurillo, 1997; Zuluaga, 2001; Mena, 2003; Timofeeva, 2009a, 2009b).

La identificación, la comprensión y el manejo de las UF desautomatizadas requiere de un dominio profundo de la dinámica fraseológica, pues es imprescindible una asimilación previa sobre cómo se configura y se articula el significado de la UF original o, recurriendo a una cita de un trabajo ya mencionado de Prodromou (2003: 46), "if one knows the way phraseology works, if one belongs to the speech community which shares the system and is defined by its cultural norms, then one can bend and break its rules and patterns...".

Por todo ello, la utilidad de este tipo de actividades se plantea en los niveles más avanzados de estudio, ya que también su finalidad va más allá de la formación de la denominada competencia fraseológica. En realidad, cuando hablamos de la desautomatización nos estamos refiriendo a estructuras que han dejado de ser fraseológicas, pero su valor discursivo se obtiene solo a partir del conocimiento de las características de la UF de origen. Por tanto, su tratamiento en clase persigue el doble objetivo de indagar en el comportamiento discursivo de las UF, por un lado, y en las posibilidades creativas que ofrece la manipulación de este tipo de unidades ${ }^{11}$.

De acuerdo con la propuesta seguida en este trabajo, también el recurso de desautomatización recibe un enfoque más coherente desde el punto de vista del modelo de significación fraseológica desarrollado, puesto que la reflexión en torno a qué parte de la información y en qué medida (dependiendo de su grado de convencionalización) se ve afectada por la alteración ofrece una visión más ordenada sobre los efectos perlocutivos buscados con este procedimiento (cfr. Timofeeva, 2012: 267-274). Por ello, a nuestro modo de ver, la inclusión en clase de actividades sobre las UF manipuladas se ajusta perfectamente al objetivo de promover el desarrollo de la capacidad metalingüística del alumno.

En definitiva, las sucintas reflexiones metodológicas desarrolladas en este apartado nos

11 En Grupo GRIALE (2011) se pueden ver algunos ejemplos de ejercicios de la desautomatización fraseológica, así como de otros procedimientos lingüísticos, para trabajar el fenómeno de la ironía en clase. 
Ilevan a reafirmar que el modelo de significación fraseológica expuesto presenta interesantes implicaciones didácticas, ya que a través de él podemos fomentar y reforzar el proceso de formación de las diversas subcompetencias que conforman la competencia comunicativa. Por otra parte, una presentación articulada y sistematizada de las informaciones de diversa índole que configuran el significado fraseológico en dos niveles de su actuación —el semántico y el pragmático - ofrece al estudiante una idea muy clara de la naturaleza y el funcionamiento comunicativo de las UF, así como le proporciona parámetros del tratamiento interlingüístico de este tipo de unidades.

\section{Conclusiones}

Llegados a este punto, y con el fin de compendiar las consideraciones vertidas a lo largo de este trabajo, podemos plantear las siguientes reflexiones finales. Como indicábamos en el apartado 2, las UF se conciben tradicionalmente desde la perspectiva de su inherente dificultad para un hablante no nativo de una lengua. Sin embargo, tal visión resulta paradójica si atendemos a la naturaleza eminentemente holística del acto comunicativo verbal. En efecto, y dadas nuestras limitaciones articulatorias, los humanos nos las hemos ingeniado para establecer una serie de rutinas inferenciales que vienen asociadas a determinadas combinaciones lingüísticas. De esta forma, conseguimos expresar más con menos, y las UF ocupan un papel especial dentro de esta caracterización holística. Por tanto, hemos afirmado que la didáctica de los fraseologismos ha de partir de esa premisa sobre su carácter natural e imprescindible y contemplar las UF no como obstáculos, sino como elementos de apoyo en la formación de la capacidad holística en una LE.

Desde esta perspectiva, cabe indagar en cómo las UF articulan su significado para comprender en qué medida dicha articulación se imbrica en el proceso de formación de la competencia comunicativa en LE. Defendemos que el significado de UF puede estructurarse con el fin de crear un modelo operativo que, según hemos expuesto en el apartado 3, aglutina una serie de datos articulados en dos niveles, el semántico y el pragmático, acorde a las características idiosincrásicas de este tipo de estructuras.

El modelo de significación fraseológica que hemos presentado se convierte en el apartado 4 en el punto de partida para el cambio de enfoque según el cual se plantea la posibilidad de orientar la formación de ciertos aspectos de la competencia comunicativa desde la fraseología, y no al revés como se ha hecho tradicionalmente. Por ello, se presentan una serie de consideraciones metodológicas en las que se basaría el diseño de actividades concretas para trabajar las diversas subcompetencias de la competencia comunicativa a partir de la fraseología. Siguiendo la línea de este trabajo, nos hemos limitado a exponer pautas y orientaciones al respecto, puesto que la elaboración de materiales docentes es de por sí una tarea compleja que sobrepasa los objetivos de este artículo.

En definitiva, en este trabajo hemos intentado mostrar nuevas vías de reflexión metodológica sobre el tratamiento didáctico de las UF en clase de LE, basadas en una concepción cualitativamente distinta de la significación fraseológica. A nuestro juicio, tales reflexiones pueden contribuir a optimizar el proceso de adquisición tanto de la fraseología como de la competencia holística en general en una LE.

\section{Bibliografía citada}

Alcón Soler, Eva, 2002: Bases Lingüísticas y Metodológicas para la Enseñanza de la Lengua Inglesa, Castellón: UJI.

Altenberg, Bengt y Mats Eeg-Olofsson, 1990: "Phraseology in Spoken English: Presentation of a Project" en Jan Aarts y Willem Meiss (eds.): Theory and Practice in Corpus Linguistics, Amsterdam: Rodopi, 1-26.

Celce-Murcia, Marianne, 2007: "Rethinking the 
Role of Communicative Competence in Language Teaching" en Eva Alcón y M. ${ }^{a}$ Pilar SAfont (eds.): Intercultural Language Use and Language Learning, Dordrecht: Springer, 41-57.

Cifuentes Honrubia, José Luis, 1994: Gramática cognitiva. Fundamentos críticos, Madrid: Eudema.

Cifuentes Honrubia, José Luis, 2003: Locuciones prepositivas: sobre la gramaticalización preposicional en español, Alicante: Universidad.

Corpas Pastor, Gloria, 1996: Manual de fraseología española, Madrid: Gredos.

Cuenca, Maria Josep y Joseph Hilferty, 1999: Introducción a la lingüística cognitiva, Barcelona: Ariel.

Detry, Florence, 2008: "Consideraciones metodológicas para el tratamiento de las expresiones idiomáticas en clase de español como lengua extranjera (ELE)", LinRed VI [http://www.linred. es/articulos.htm\#d, fecha de consulta: I de junio del 2012].

DFDEA = Manuel SeCo y otros, 2004: Diccionario fraseológico documentado del español actual, Madrid: Aguilar.

DFEM = Fernando VARELA y Hugo KUBARTH, 1994: Diccionario fraseológico del español moderno, Madrid: Gredos.

Dobrovol'SkIJ, Dmitrij, 1990: "Tipologuija idiom” en Veronika Telıja y otros (eds.): Frazeografija $v \mathrm{Ma}$ sinnom fonde russkogo jazyka, Moskva: Nauka.

Dobrovol'SkIJ, Dmitrij, 1997: “Natsional'no-kulturnaja specifika vo fraseologuii(I)", Voprosy jazykoznanija 6, 37-48.

Dobrovol'SKIJ, Dmitrij, 1998: “Natsional'no-kulturnaja specifika vo fraseologuii(II)", Voprosy jazykoznanija 6, 48-57.

Dobrovol'SkIJ, Dmitrij y Elisabeth PIIRAInen, 2005: Figurative language: cross-cultural and crosslinguistic perspectives, Amsterdam: Elsevier.

DRAE $=$ Real Academia Española, 2001: Diccionario de la lengua española (22. ${ }^{a}$ ed.), Madrid: Espasa.

DUE = María Moliner, 1998: Diccionario de uso del español, Madrid: Gredos.

Forment Fernández, M. ${ }^{a}$ del Mar, 1998: "La didáctica de la fraseología ayer y hoy: del aprendizaje memorístico al agrupamiento en los repertorios de funciones comunicativas" en Francisco Moreno y otros (eds.): La enseñanza del español como lengua extranjera: del pasado al futuro. Actas del VIII Congreso Internacional de ASELE, Alcalá de Henares: Universidad, 339-347.

Grupo Griale, 2011: ¿Estás de broma? 20 actividades para practicar la ironía en clase de ELE, Madrid: Edinumen.

Hymes, Dell, 1995 [1971]: "Acerca de la competencia comunicativa" en Miquel Llobera y otros (eds.): Competencia comunicativa. Documentos básicos en la enseñanza de lenguas extranjeras, Madrid: Edelsa, 27-47.

Kabakova, Svetlana, 2002: Obraznoje osnovanije idiom. Psijolingvokulturologuicheskije aspekty, München: Otto Sagner.

Kress, Gunther, 2000: "Multimodality: Challenges to Thinking About Language", TESOL Quarterly 34.2, 337-340.

Lee, Seowon, 2004: "Teaching lexis to EFL students: a review of current perspectives and methods", Annual Review of Education, Communication and Language Sciences, Volume 1 , [http://research.ncl.ac.uk/ARECLS/vol__documents/Seowon/Seowon.htm, fecha de consulta: 20 de mayo del 2012].

Lennon, Paul, 1998: "Approaches to the Teaching of Idiomatic Language", IRAL, Vol. XXXVI/1, 11-30.

LEONTARIDI, Eleni y otros, 2008: "Las unidades fraseológicas del español: su enseñanza y adquisición en la clase de ELE" en Jornadas de Formación del Profesorado en la Enseñanza de ELE y la Literatura Española Contemporánea, 187-206 
[http://invenio.lib.auth.gr/record/125539/, fecha de consulta: 20 de mayo del 2012].

Levinson, Stephen, 1995: "Three levels of meaning" en F. R. Palmer (ed.): Grammar and meaning, Cambridge: University Press, 90-115.

Levinson, Stephen, 2004 [2000]: Significados presumibles: la teoría de la implicatura conversacional generalizada, Madrid: Gredos.

Llobera, Miquel y otros, 1995: Competencia comunicativa. Documentos básicos en la enseñanza de lenguas extranjeras, Madrid: Edelsa.

MCER = Consejo De Europa, 2002: Marco Común Europeo de Referencia para las Lenguas: aprendizaje, enseñanza, evaluación, Madrid: Instituto Cervantes-MECD, Anaya.

Mena Martínez, Flor, 2003: "En torno al concepto de desautomatización fraseológica: aspectos básicos", Tonos digital 5, [http://www.um.es/tonosdigital/znum5/estudios/H-Edesautomatizacion. htm, fecha de consulta: 20 de mayo del 2012].

Olímpio de Olivelra, M. ${ }^{a}$ Eugenia, 2006: "Fraseología y enseñanza de español como lengua extranjera”, RedELE 5 [http://www.educacion.gob.es/redele/Biblioteca-Virtual/2006/memoriaMaster/1Semestre/OLIMPIO-D.html, fecha de consulta: 15 de mayo del 2012].

Pawley, Andrew y Frances Hodgetts Syder, 1983: "Two Puzzles for Linguistic Theory: Nativelike Selection and Nativelike Fluency" en Jack C. Richards y Richard W. Schmidt (eds.): Language and Communication, London: Longman, 191-226.

Prodromou, Luke, 2003: "Idiomaticity and the nonnative speaker", English Today 74, vol. 19, n² 2, 4248.

Prodromou, Luke, 2008: English As a Lingua Franca: A Corpus-Based Analysis, London: Continuum International Publishing Group.

Ruiz Gurillo, Leonor, 1997: "Relevancia y fraseología: la desautomatización en la conversación co-
Ioquial”, Español Actual 68, 21-30.

Ruiz Gurillo, Leonor, 1999: "Un enfoque didáctico de la fraseología española para extranjeros" en M. ${ }^{a}$ José Coperías y Julia Sanmartín (coords.): Quaderns de Filologia. València: Universitat.

Ruiz Gurillo, Leonor, 2001: Las locuciones en español actual, Madrid: ArcoLibros.

SinclaIR, John M. (ed.), 1987: Looking Up, London: Collins.

SinclaiR, John M., 1991: Corpus, Concordance, CoIlocation, Oxford: University Press.

Solano Rodriguez, M. a Ángeles, 2004: Unidades Fraseológicas francesas. Estudio en un corpus: la Pentalogía de Belleville de Daniel Pennac. Planteamiento didáctico. Tesis doctoral. Murcia: Universidad [http://www.cervantesvirtual.com/ FichaObra.html?Ref=14551\&ext=pdf\&portal=177, fecha de consulta: 20 de abril del 2012].

TelIJA, Veronika y otros (eds.), 1990: Frazeografija v Masinnom fonde russkogo jazyka, Moskva: Nauka.

TelıA, Veronika, 1996: Russkaja fraseologuija, Moskva: Shkola "Yazyki russkoj kultury".

Timofeeva, Larissa, 2009a: "Las unidades fraseológicas" en Leonor Ruiz Gurillo y Xose Padilla (eds.): Dime cómo ironizas y te diré quién eres: una aproximación pragmática a la ironía, Frankfurt am M.: Peter Lang, 193-217.

Timofeeva, Larissa, 2009b: "La desautomatización fraseológica: un recurso para crear y divertir" en Juan Luis Jiménez Ruiz y Larissa Timofeeva (eds.): Estudios de Lingüística: Investigaciones lingüísticas del siglo XXI, Alicante: Universidad, 249-271. TimofeEva, Larissa, 2012: El significado fraseológico. En torno a un modelo explicativo y aplicado, Madrid: Ediciones Liceus.

Traugott, Elizabeth C. y Richard B. Dasher, 2002: Regularity in Semantic Change, Cambridge: University Press.

Wood, David, 2004: "An Empirical Investigation 
into the Facilitating Role of Automatized Lexical Phrases in Second Language Fluency Development", Journal of Language and Learning, vol. 2, $n^{\circ} 1,27-50$.

Wotjak, Gerd, 1983: "En torno a la traducción de unidades fraseológicas (con ejemplos tomados del español y el alemán)", Linguistische Arbeitsberichte 40, 56-80.

Zuluaga, Alberto, 2001: "Análisis y traducción de las UF desautomatizadas", PhiN 16, 67-83 [http:// www.fu-berlin.de/phin/phin16/p16t5.htm, fecha de consulta: 20 de junio del 2012]. 